\title{
A Search for Candidate Li-Rich Giant Stars in SDSS DR10
}

\author{
Duane F. Carbon \\ NASA Ames Research Center, NASA Advanced Supercomputing Facility, Moffett Field, CA, \\ 94035-1000, USA; \\ Address correspondence to: Duane.F.Carbon@nasa.gov \\ Richard O. Gray \\ Appalachian State University, Dept. of Physics and Astronomy, CAP Bldg., Boone, NC \\ 28608-0001; \\ Bron C. Nelson \\ NASA Ames Research Center, NASA Advanced Supercomputing Facility, Moffett Field, CA, \\ 94035-1000, USA; \\ and \\ Christopher Henze \\ NASA Ames Research Center, NASA Advanced Supercomputing Facility, Moffett Field, CA, \\ 94035-1000, USA
}

\begin{abstract}
We report the results of a search for candidate Li-rich giants among 569,738 stars of the SDSS DR10 dataset. With small variations, our approach is based on that taken in an earlier search for EMP/CEMP stars and uses the same dataset. As part of our investigation, we demonstrate a method for separating post-main sequence and main sequence stars cooler than $T_{\text {eff }} \approx 5800 \mathrm{~K}$ using our feature strength measures of the Sr II 4078, Fe I 4072, and Ca I 4227 lines. By taking carefully selected cuts in a multidimensional phase space, we isolate a sample of potential Li-rich giant stars. From these, using detailed comparison with dwarf and giant MILES stars, and our own individual spectral classifications, we identify a set of high likelihood candidate Li-rich giant stars. We offer these for further study to promote an understanding of these enigmatic objects.
\end{abstract}

Subject headings: techniques: spectroscopic — stars: chemically peculiar — methods: data analysis - surveys 


\section{INTRODUCTION}

The many aspects of the evolution of the Li abundance from the Big Bang to the present has generated a very large and complex literature. The existence of Li-rich giants, first discovered by Wallerstein and Sneden (Wallerstein \& Sneden 1982), is one part of the Li story. The origin of these unexpected stars is still not fully understood. Here we outline the main points needed to appreciate the strangeness of Li-enriched post-main sequence (post-MS) stars. For those desiring a deeper review, we recommend the useful and detailed summaries of the observed and predicted postMS evolution of the Li abundance given by Brown et al. (1989); Ruchti et al. (2011); Casey et al. (2016) and the references cited therein.

The maximum $\mathrm{Li}$ abundances of $\mathrm{F}$ and $\mathrm{G}$ main sequence $(\mathrm{MS})$ stars range from $\mathrm{A}(\mathrm{Li}) \approx 2.11$ for ancient, low metallicity stars all the way up to $\mathrm{A}(\mathrm{Li}) \approx 3.3$ for young, metal rich stars (Lambert \& Reddy 2004; Prantzos et al. 2017). The Li abundance is expected to change dramatically once stars leave the main sequence. Classical stellar evolution theory predicts that the $\mathrm{Li}$ abundance will monotonically decrease once the bottom of the outer convective envelope begins to move inward during post-MS evolution. As the envelope deepens, it increasingly entrains mass zones where complete Li destruction has occurred. This in turn dilutes the MS surface abundance of Li. By the time a star reaches the base of the giant branch, the Li abundance is expected to be only $\approx 5-10 \%$ of its MS value (Iben $1967 \mathrm{a}, \mathrm{b})$. As the star ascends the giant branch in the classical models, the convective envelope continues to deepen and the Li abundances are predicted to drop even further. Moreover, observational evidence (e.g., Brown et al. 1989; Mallik 1999; Liu et al. 2014) indicates that the actual Li depletion is substantially greater than the classical models predict for the majority of $\mathrm{G}$ and $\mathrm{K}$ giants.

With this background, it was a great surprise when Wallerstein and Sneden in 1982 reported the discovery of a metal-rich, field K giant, HD 112127, whose Li I 6708 resonance doublet line had an equivalent width of $0.45 \AA$. A model atmosphere abundance analysis of the weaker Li I 6104 transition led to $\mathrm{A}(\mathrm{Li}) \approx 3.0$, very substantially higher than expected theoretically. Kraft et al. (1999) reported the first Li-rich giant in a globular cluster, a star on the first ascent of the giant branch in $\mathrm{M} 3$, with $\mathrm{A}(\mathrm{Li}) \approx 3.0$ and a $\mathrm{Li} \mathrm{I} 6708$ equivalent width of $0.52 \AA$. Over the years, other Lirich giants have been found, both in Population I and Population II. These stars are quite rare however, comprising $\leq 1 \%$ of giant stars (Brown et al. 1989; Ruchti et al. 2011; Liu et al. 2014; Kirby et al. 2016). The recent tabulation by Casey et al. (2016) lists only 127 known giant stars with $\mathrm{A}(\mathrm{Li})>2.0$; the values of $\mathrm{A}(\mathrm{Li})$ in this listing range all the way up to $\mathrm{A}(\mathrm{Li})=4.55$. The temperatures of these known Li-rich evolved stars span a considerable range: the hottest is HD 172481, an F supergiant with $T_{\text {eff }} \approx 7250 \mathrm{~K}$ (Revniers \& Van Winckel 2001) and the coolest is IRAS 125567731, an M giant with $T_{\text {eff }} \approx 3460 \mathrm{~K}$ (Alcalá et al. 2011). Most known examples, however, are found among the $\mathrm{G}$ and $\mathrm{K}$ stars where our search is optimized.

\footnotetext{
${ }^{1} \mathrm{~A}(\mathrm{Li})=\log _{10}\left(N_{L i} / N_{H}\right)+12$ is the logarithmic abundance of $\mathrm{Li}$ on a scale where $\mathrm{A}(\mathrm{H})=12.0$
} 
Numerous hypotheses have been put forward to explain these rare Li-rich giants. The majority appeal to some form of "extra" stellar mixing on the giant or asymptotic giant branches that manages to incorporate the Cameron-Fowler process (Cameron \& Fowler 1971) to generate ${ }^{7} \mathrm{Li}$. Other scenarios rely on acquiring Li-rich material from a companion. The reader is referred to Kirby et al. (2016) for a brief but insightful review of all these mechanisms.

There is a need to find as many of these rare stars as possible to get the full parameter space decribing their occurence and begin to narrow down the numerous possibilities for producing them. One of the most successful efforts in finding new Li-rich giants was that undertaken by Martell and Schetrone (M\&S13) (Martell \& Shetrone 2013). They searched the Sloan Digital Sky Survey Data Release 7 (SDSS DR7) (Abazajian et al. 2009) stars for candidates and identified 27 new Li-rich giants, 23 of which they then subjected to high resolution abundance analysis. It is the purpose of the current paper to find additional Li-rich giant candidates by carrying out a search using the data of SDSS DR10 (Ahn et al. 2014). The DR10 dataset of optical stellar spectra is roughly 1.6X larger than that of the DR7 dataset. This means that there are a substantial number of additional stars now available to examine for Li-enhancement. We carry out our investigation using a slight variation of the approach we employed earlier in a search for extremely metal-poor (EMP) stars (Carbon et al. 2017) (CHN17).

In Section 2, we briefly describe how we processed the DR10 dataset through our reduction pipeline so that we could extract the individual feature measurements that are the basis of our approach. In the subsections of Section 3, we detail how we chose our initial Li-rich candidates. In particular, we describe how we selected (Section 3.1) and tested (Section 3.2) feature measurements to impose temperature and luminosity constraints, and how we extracted (Section 3.3) a coarse sample of candidate Li-rich post-MS stars using a set of cuts in a multi-dimensional phase space. Next we describe how we refined the sample to select only the most likely candidates (Section 3.4) and then carried out a detailed spectral classification of these stars (Section [3.5). In Section 4 we discuss our final list of candidate post-MS Li-rich giants. Our principal results are summarized in Section 5 ,

\section{THE DATASET}

In our search for Li-rich giants, we use the previously prepared dataset (CHN17) composed of calibrated optical fluxes and associated data for 569,738 unique stellar spectra drawn from SDSS DR10. The associated data includes each star's coordinates, heliocentric radial velocity, median S/N, pixel-by-pixel inverse-variance values, and $u, g, r, i, z$ point spread function magnitudes (psfMag). The reader is referred to Section 2 of CHN17 for details concerning the selection of the stellar data from the whole SDSS DR10 database, the processing of the stellar fluxes through our data reduction pipeline, and the feature strength measurements that were subsequently made from the spectra. Here, we review briefly only the salient points needed for understanding the arguments in the current paper. 
The first step in our pipeline was establishing a continuum for each spectrum. Once the continuum level was established, it was possible to compute quantitative measures for the spectral lines in each spectrum. Two types of feature measures were adopted. The first was $S\left(\lambda_{i}\right)$ which is the fractional depth relative to the interpolated local continuum of an individual spectral feature at wavelength $\lambda_{i}$. The second was $D\left(\lambda_{i}\right)$ which is the depth of the line at $\lambda_{i}$ in units of the local noise level in the spectrum as determined from the spectrum's pixel-by-pixel inverse-variance values. $S\left(\lambda_{i}\right)$ is a direct measure of the line's strength while $D\left(\lambda_{i}\right)$ gives a handy measure for the line's strength relative to the local noise level. The latter can be particularly helpful when dealing with intrinsically weak lines like the Li I 6708 line central to this paper. Measurements of $S\left(\lambda_{i}\right)$ and $D\left(\lambda_{i}\right)$ were made for 1659 spectral features (each with a unique $\lambda_{i}$ ) for all the 569,738 spectra of our dataset. This produced the final dataset of nearly 2 billion feature measures used in this study.

In the CHN17 study, stars with specific interesting characteristics were isolated from the above SDSS DR10 dataset by using linked scatter plot (LSP) tools implemented on the NASA Advanced Supercomputing hyperwall. (See CHN17, Section 1.1 for a detailed description of LSPs and the hyperwall.) For example, by making judiciously selected cuts in successive 2-D phase spaces, CHN17 were able to extract numerous candidate extremely metal poor (EMP) stars from the general dataset. Because of its flexibility, the LSP method has powerful explorative capability. For this reason, we used LSPs on the hyperwall to make the initial reconnaissance of the Li-rich giant problem. It quickly became apparent that there were indeed stars with strong Li I 6708 lines in the DR10 dataset. However, because the 569,738 spectra of the dataset include a very wide range of temperatures, luminosities, and compositions, we needed to determine how to extract candidate Li-rich giants from the rest of the stars. In the next section, we explain how we accomplished this.

\section{EXTRACTING THE CANDIDATE LI-RICH GIANTS}

We require an approach which will effectively separate the relevant post-MS stars from MS stars which may have comparable Li line strengths. The domain of chief interest is that occupied by the late $\mathrm{G}$ and $\mathrm{K}$ giants. We need to select feature measures, $S(\lambda)$ and $D(\lambda)$, in our dataset that can be used to isolate stars in this desired temperature and luminosity range. Note that our approach relies solely on our feature measures. We specifically chose not to employ SDSS-provided quantities such as $T_{\text {eff }}$ and $\log g$ simply because of the large errors that can occur in individual values of these quantities (e.g., M\&S13, Appendix A). The following subsections detail how we used the feature measures to arrive at a list of Li-rich giant candidates.

\subsection{MILES spectra to establish temperature and luminosity constraints}

Gray \& Corbally (2009) note that the Ca I 4227 line progressively strengthens with decreasing temperature in going from $\mathrm{G}$ through $\mathrm{K}$ spectral types, while the hydrogen lines progressively 
weaken over the same spectral range. This suggests that $S(\mathrm{Ca}$ I 4227$)$ or $S(\mathrm{H} \mathrm{I})$ could be used as a first-order surrogate for stellar temperature. (We note here that we investigated using colors based on the SDSS $u, g, r, i, z$ magnitudes as temperature surrogates but found that they did not lead to clean separation between MS and post-MS stars.) In order to estimate luminosity in G and K star domain, Gray \& Corbally (2009) and White et al. (2007) suggest a number of possible metal line strengths and ratios. To determine whether any of these might be helpful in our investigation, we turned to the MILES spectrum library (Sánchez-Blázquez et al. 2006) .

The 985 stars in the MILES library were selected for the purposes of stellar population synthesis. As a result, they cover a wide range of temperatures, luminosities, and metallicities with particularly good coverage for the spectral ranges of most interest to us (e.g., Sánchez-Blázquez et al. 2006, Figure 1). The MILES spectra span the whole SDSS optical wavelength range relevant to our study and have essentially the same spectral resolution as the SDSS spectra. Moreover, the stars in this library have carefully researched $T_{\text {eff }}, \log g$, and metallicity $([\mathrm{Fe} / \mathrm{H}])$ drawn from the literature (Cenarro et al. 2007). These attributes make the MILES spectra ideal for determining which luminosity criteria might be most effective for separating G and K MS stars from post-MS stars. To take advantage of the MILES library, we ran the entire dataset of nearly 1000 MILES spectra through the same spectral reduction pipeline that we used in our earlier study. The pipeline computed continua for each of the MILES spectra and then computed $S(\lambda)$ and $D(\lambda)$ measures for each of the 1659 spectral features we use. Details of the pipeline process may be found in CHN17, Sections 2.1-2.3.

To explore which of the Gray-Corbally and White et al. luminosity criteria might be best for our purposes, we extracted two subsets from our full MILES dataset of feature measurements. The first subset, which we used to represent MS stars, was comprised of the 344 MILES A through $\mathrm{K}$ stars with Cenarro et al. (2007) $\log g>3.80$. The second subset, representing post-MS stars, was comprised of the 254 MILES A through K stars with Cenarro et al. (2007) $\log g \leq 3.80$. The division in $\log g$ was chosen to be comparable to that adopted by M\&S13 in isolating post-MS stars for their study.

Many of the Gray-Corbally and White et al. luminosity criteria in the G-K spectral range are ratios of lines strengths (or sums of line strengths), e.g., the ratio of Y II 4375 to Fe I 4384. We represented these by taking the ratios of the corresponding line strength measures, as in $S$ (Y II 4375)/S(Fe I 4384). Using the MS and post-MS subsets of MILES data, we examined the various luminosity sensitive line ratios versus the feature strengths of the likely temperature sensitive lines: $S(\mathrm{H} \alpha), S(\mathrm{H} \beta), S(\mathrm{H} \gamma)$, and $S(\mathrm{Ca}$ I 4227). After considerable experimentation, we found that $S$ (Sr II 4078)/S(Fe I 4072) vs $S$ (Ca I 4227) gave the clearest separation between MS and post-MS stars for the G-K stars. We show this separation in Figure 1. The luminosity sensitivity of the $S(\mathrm{Sr}$ II 4078)/S(Fe I 4072) ratio is a result of the rather different electron pressure sensitivities of these two lines in the cooler stars. The line ratio systematically shifts as the gravity, and hence electron pressure, decreases with increasing luminosity. A discussion of such effects may be found in Gray (1992), for example. 


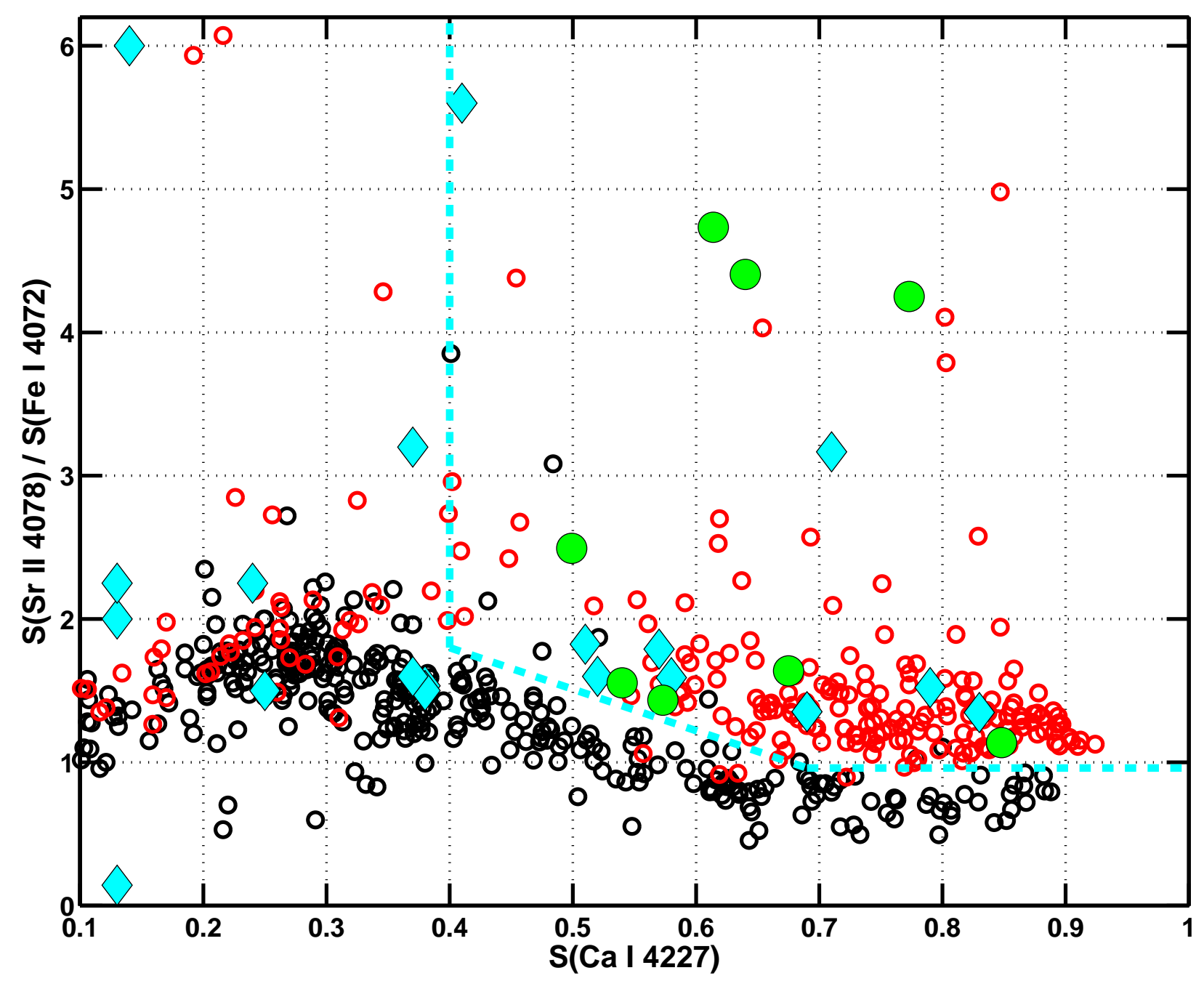

Fig. 1.- Selected luminosity indicator, $S(\mathrm{Sr}$ II 4078)/S(Fe I 4072), versus $S$ (Ca I 4227), a temperature surrogate. The MILES MS stars are plotted as black circles and the post-MS stars as red circles. See text for details of how the MILES stars were divided according to log $g$. The M\&S13 stars are plotted as cyan filled diamonds. The region of this phase space where we searched for candidate Li-rich post-MS stars lies to the right of the vertical cyan dashed line at $S$ (Ca I 4227$)=0.4$ and above the lower bound defined by the cyan dashed lines running to the right. The candidate Li-rich stars that we identify in Table 1 are displayed in this figure as green filled circles. 
The locations of the MS and post-MS stars (black and red circles, respectively) in Figure 1, show that, while there are a few exceptions, the Sr II/Fe I ratio nicely discriminates between MS and post-MS stars in the region $S(\mathrm{Ca}$ I 4227$) \geq 0.4$. In contrast, the Sr II/Fe I ratio becomes unreliable as a luminosity diagnostic for $S(\mathrm{Ca}$ I 4227$)<0.4$. Examination of the $S(\mathrm{Ca}$ I 4227$)$ vs $T_{\text {eff }}$ relation for the MILES stars indicates that the $S(\mathrm{Ca}$ I 4227$)=0.4$ boundary occurs at $\approx 5800 \mathrm{~K}$, a temperature that corresponds to early-mid G stars in the case of dwarfs (Boyajian et al. 2012). Thus, since it places us in the stellar temperature range most relevant to our search, restricting our search to stars with $S$ (Ca I 4227$) \geq 0.4$ should pose no difficulty. However, in the next subsection, we will note one important caveat.

\subsection{Comparison with confirmed Li-rich giants}

It is helpful to illustrate how a known set of Li-rich giants are distributed in Figure 1, M\&S13 searched for Li-rich giants among the stars of SDSS DR7. They chose a set of 8535 stars from DR7 whose SEGUE Stellar Parameter Pipepine (SSPP) $T_{\text {eff }}$ and $\log g$ values indicated that they would be red giant branch (RGB) stars lying somewhere between slightly below the red giant bump and the red giant tip. They estimated the Li 6708 strength in these stars using a spectral index they computed centered on the line. Selecting only those stars with the most promising $\mathrm{Li}$ spectral indices (162 stars), they used low resolution spectrum synthesis to sub-select a set of 36 for follow-up high resolution study. Of these 36, they confirmed that 27 were indeed Li-rich based on high resolution spectroscopy and spectrum synthesis. (We note that M\&S13 present derived Li abundances for only 23 of the 27 stars because of S/N problems. Nevertheless, we will consider all 27 as "confirmed Li rich" as indicated in their Table 1.) Of these 27 SDSS DR7 Li-rich stars, 19 are included in the download for our DR10 dataset, the 8 missing stars violated one or more of the selection criteria we adopted in selecting the stars for our dataset.

In Figure 1 we show the 19 M\&S13 stars as cyan diamonds. We see that 9 of the 19 M\&S13 stars fall comfortably in the region occupied by post-MS stars with $S$ (Ca I 4227) $\geq 0.4$. The remainder, with smaller $S(\mathrm{Ca}$ I 4227) values, fall in the region where the $S(\mathrm{Sr}$ II 4078)/S(Fe I 4072) ratio does not reliably distinguish luminosities. Eight of the 10 stars in this latter region have $[\mathrm{Fe} / \mathrm{H}]$ determined by M\&S13 (their Table 2). Six have $[\mathrm{Fe} / \mathrm{H}]$ ranging from -1.4 to -2.6 , i.e., they are metal poor. The other 2 stars have the highest $T_{\text {eff }}$ values $(5250 \mathrm{~K}, 5625 \mathrm{~K})$ of the stars with $S(\mathrm{Ca}$ I 4227$)<0.4$ as well as depressed $[\mathrm{Fe} / \mathrm{H}](\leq-0.29)$. (The 2 stars that do not have M\&S13 $[\mathrm{Fe} / \mathrm{H}]$ values, have SDSS $[\mathrm{Fe} / \mathrm{H}]$ of -0.66 and -1.50 .) Both low metallicity and higher $T_{\text {eff }}$ could explain the presence of M\&S13 stars to the left of the $S(\mathrm{Ca}$ I 4227$)=0.4$ boundary. All of the stars to the right of the $S(\mathrm{Ca} \mathrm{I} 4227)=0.4$ boundary have $[\mathrm{Fe} / \mathrm{H}]$, as determined by M\&S13, ranging from -0.47 to +0.41 . This suggests that, by restricting ourselves to stars with $S(\mathrm{Ca}$ I 4227$) \geq 0.4$, we may run the risk of missing Li-rich giants with low metallicities. Since we see no obvious way at this time to devise a luminosity criterion that does not risk excluding such stars, we shall proceed. Low metallicity giants that are sufficiently cool (hence having intrinsically stronger Ca I 4227) 
might still land to the right of the $S(\mathrm{Ca}$ I 4227$)=0.4$ boundary and thus be detectable by us.

\subsection{Imposing the final constraints}

In order to arrive at a useful set of Li-rich giant candidates it is necessary to constrain more than just the value of $S(\mathrm{Li} \mathrm{I} 6708)$ and the region in $S(\mathrm{Sr}$ II 4078)/ $S($ Fe I 4072) vs $S(\mathrm{Ca}$ I 4227) space. For good quality results, one must also add constraints on the noise levels both overall and locally in the $6700 \AA$ and $4070 \AA$ regions. Similarly, it is necessary to guard against TiO contamination of the Li I 6708 line region. After considerable experimentation, we chose the set of constraints listed below. The additional constraints eliminated many spectra in which noise/contamination produced uncertain results for the value of $S(\mathrm{Li}$ I 6708) and/or the $S(\mathrm{Sr}$ II 4078)/S(Fe I 4072) ratio.

The measures we selected and the constraints we imposed on them are summarized in the logical expressions below. All of these constraints, 1 - 6, are applied at the same time to the feature measures of the 569,738 stars in the dataset. Only stars that simultaneously satisfy all the specified constraints are considered in the remaining discussion.

$$
\begin{aligned}
& \begin{array}{r}
(0.4 \leq S(\mathrm{Ca} \text { I } 4227)<0.69) \&((S(\text { Sr II } 4078) / S(\text { Fe I } 4072)) \geq(2.96-2.90 * S(\mathrm{Ca} \\
(S(\mathrm{Ca} \text { I } 4227) \geq 0.69) \&(0.96<(S(\text { Sr II } 4078) / S(\text { Fe I } 4072)))
\end{array} \\
& S(\text { Li I } 6708) \geq 0.04 \\
& \operatorname{median}(\mathrm{S} / \mathrm{N})>20 \\
& D(\operatorname{Li} \text { I } 6708)>1.0 \\
& S(S \text { r II 4078) }>0.0 \\
& S(\mathrm{TiO} 6815)<S(\mathrm{Li} \text { I } 6708)
\end{aligned}
$$

We now briefly describe the rationale for each cut shown above:

Constraints 1a and 1b apply the luminosity discriminant ratio $S$ (Sr II 4078)/S(Fe I 4072) described in Section 3.1. Constraint 1a applies to the left-hand portion of the region outlined by cyan dashed lines in Figure 1 (i.e., $0.4 \leq S(\mathrm{Ca}$ I 4227) $<0.69$ and above the sloping cyan dashed line); 1 b applies to the right-hand portion (i.e., $S$ (Ca I 4227) $\geq 0.69$ and above the horizontal cyan dashed line).

Constraint 2 further selects out those stars which have Li I absorption strengths above a minimum threshold. We selected the threshold to be equal to the $S$ (Li I 6708) measure of the M\&S13 Li-rich giant with the weakest Li I feature. 
Constraint 3 isolates the objects which have sufficient $\mathrm{S} / \mathrm{N}$ in their spectra to make estimating luminosity and Li I strength more robust. We found that the spectra of stars with poorer $\mathrm{S} / \mathrm{N}$ are generally much too noisy to yield reliable measures of either $S(\mathrm{Sr} I \mathrm{II}) / S(\mathrm{Fe} \mathrm{I})$ or $S(\mathrm{Li} \mathrm{I})$.

Constraint 4 further limits the subset of stars to those with Li I 6708 line depths more than $1 \sigma$ above the local noise level. This helps eliminate stars that have excessive noise near the Li I line.

Constraint 5 limits the stars to only those with detected Sr II 4078 absorption, removing stars for which random noise, or poor continuum placement, produces a false emission feature.

Constraint 6, which uses a ${ }^{48} \mathrm{Ti}^{16} \mathrm{O}(3,2)$ gamma system band head, was introduced to bias against stars for which the $\mathrm{TiO}$ bands were becoming sufficiently strong that they were noticeably affecting the region of the Li I line.

To impose the constraints described above, we constructed a suite of MATLABC) (MATLAB 2011) codes implemented on a single computer workstation. The suite reads in the constraints on specified feature variables and returns a list of those stars for which the specified variables simultaneously satisfy all the constraints. This is logically equivalent to the CHN17 method of making a series of successive cuts in 2-D phase spaces that was the basis of the LSP approach. Applying the constraints 1 through 6 to the dataset of 569,738 SDSS DR10 stars produces a subset of 1,523 stars which are potentially Li-rich giants. In the next sub-section, we will describe how we select out the most likely candidate Li-rich giants.

\subsection{Extracting the Candidate Li-rich Giants}

\subsubsection{Eliminating the obvious false positives}

The feature constraint on median $\mathrm{S} / \mathrm{N}$ in Equation (3) was intentionally left "softer" than it might have been so as to capture as many candidates as possible. However, this means that stars may slip through the constraints whose spectra are too contaminated by noise in crucial spectral regions to be sure of their status. In addition, some of the feature strengths used in the constraints may have erroneous values caused by poor continuum placement. (A more detailed discussion of these issues may be found in CHN17, Section 4.) We dealt with these issues by visually examining the spectra of each of the 1,523 stars selected by the constraints of the previous section. The visual examination was done in two steps.

In the first step, the chief criteria were the strength and apparent position of the purported Li I line, whether the spectral regions around Li I line and the luminosity indicators appeared relatively unaffected by noise, whether there appeared to be $\mathrm{TiO}$ contamination of the Li region, and whether the continuum placement was appropriate. A secondary consideration was whether the

Li I 6708 line was comparable to or stronger than Ca I 6718 line (see Casey et al. (2016, Figure 5)). 
The ratio of these two lines was used by Kumar et al. (2011) in their study to identify candidate Li-rich stars from low-resolution giant spectra. This coarse initial cull was straightforward and was accomplished relatively quickly. It eliminated 1,350 stars from further consideration, the vast majority because the local noise level was too large to be confident of the Li line strength.

In the second step, the spectra of the 173 remaining stars were subjected to a more prolonged and careful visual inspection which concentrated on the position, shape, and strength of their Li I 6708 line and the quality of the spectrum. Stars were eliminated if the apparent Li I feature appeared to be strongly asymmetric, shifted significantly from its nominal position, or was too similar in appearance to the surrounding noise features. This second visual cull left 49 candidates. These 49 stars included all 9 of the M\&S13 Li-rich giants which fell into our search region, evidence that our selection procedure was working well. The next sub-sections describe how we confirmed whether the final 40 previously unrecognized Li-rich candidates were indeed giants.

\subsubsection{Comparison with MILES stars}

To increase our confidence in the likelihood that we were selecting stars that were good Li-rich giant candidates, we first carried out a systematic comparison of each of the 40 stars with the MILES MS and post-MS stars. First we normalized the spectrum of each Li-rich giant candidate and each MILES MS and post-MS star. This was accomplished by normalizing each spectrum by its continuum and then by its flux at $5837 \AA$ so as to keep the scales of the different spectra consistent. Next we interpolated the resulting MILES spectra onto the SDSS DR10 wavelength set over the interval [3850 - $7400 \AA]$. Using the resulting fluxes, we computed the following summed square differences, SSD, for each of the 40 stars against each of the spectra of all the MILES MS stars seriatim and then, separately, all the MILES post-MS stars:

$$
\mathrm{SSD}=\sum_{k=1}^{n}\left[F_{\text {cand }}\left(\lambda_{k}\right)-F_{\mathrm{MILES}}\left(\lambda_{k}\right)\right]^{2},
$$

where $F_{\text {cand }}\left(\lambda_{k}\right)$ is the normalized flux at wavelength $\lambda_{k}$ of one of the candidate Li-rich stars, $F_{\text {MILES }}\left(\lambda_{k}\right)$ is the corresponding normalized flux of one of the MILES stars, and $\mathrm{n}$ is the number of wavelengths in the common wavelength set.

For each candidate Li-rich star, we compared its spectrum with the closest matching (i.e., smallest SSD values) MILES MS and post-MS stars to see whether the candidate spectrum appeared more consistent with the spectra of dwarfs or giants. Attention was paid not only to the Sr II 4078/Fe I 4072 ratio, but also to the strengths of Sr II 4078 relative to the Fe I 4064 and Fe I 4046 lines (Gray \& Corbally 2009). We also considered the values of SSD for the candidate star and the ten closest matches from the MILES main sequence and post-MS lists. For many stars, the SSD values for the top ten closest matches were very strongly in favor of a candidate being most like a giant or dwarf. 
Based on the above comparisons, 31 stars were rejected because they more closely matched the spectra of MILES MS stars both in their Sr II to Fe I line ratios and in their SSD values. Nine stars remained as candidates to be Li-rich giants. We decided it was prudent to subject these 9 stars to an additional final check. The next sub-section describes the effort by one of us (ROG) to examine the spectra of the 9 stars in detail and make definitive spectral type classifications based on more than the limited number of spectral features we have considered up to this point.

\subsection{Detailed spectral classification}

While the SDSS spectra have a much larger spectral range, the most sensitive temperature and luminosity criteria are found in the violet - green region, $3800-5600 \AA$. Because of the unavailability of an MK standard star library for the SDSS spectra, we convolved the SDSS spectra with a gaussian to reduce the resolution to that of the libnor36 MK Standards library 2 (3.6 $/ 2$ pixels) of Gray \& Corbally (2014).

Gray \& Corbally (2009) detail the temperature and luminosity criteria used in the MK classification of G- and K-type stars. In summary, temperature criteria involve the ratio of low-excitation neutral metal lines to hydrogen lines (Fe I $\lambda 4046 / \mathrm{H} \delta$, Fe I $\lambda 4144 / \mathrm{H} \delta$, Fe I $\lambda 4383 / \mathrm{H} \gamma$ as well as similar line ratios in the vicinity of $\mathrm{H} \beta$ ). Those ratios, however, are invalid in metal-poor stars, and in that case, the ratio of lines of the Cr I triplet $(\lambda \lambda 4254,4275,4290$ - all resonance transitions) with the higher-excitation Fe I $\lambda \lambda 4250,4260$, and 4326 lines provide metallicity-independent temperature criteria. Luminosity criteria include the ratios of $\mathrm{Sr}$ II $\lambda 407 \sqrt{3}$ to nearby Fe I lines ( $\lambda \lambda 4046,4064$, and 4072), Sr II $\lambda 4216 / \mathrm{Ca}$ I $\lambda 4226$, Y II $\lambda 4376 / \mathrm{Fe}$ I $\lambda 4383$ as well as the strength of $\mathrm{CN}$ violet system, in particular the band blueward of the $\lambda 4215$ bandhead. However, in stars with carbon abundance peculiarities, the $\mathrm{CN}$ band strength can give spurious results, as proved to be the case with a number of stars in the candidate Li-rich sample under consideration.

The spectral types were determined by eye on the computer screen by direct comparison with the libnor36 MK standards. The spectral types we obtained are listed in Table 1 for the 8 candidates which proved to be giants. One candidate (J215914.37+004515.8) turned out to be a G9 dwarf and will not be considered further. Three out of the final 8 appear to be normal late Gand early K-type giants. The remaining stars, all late G- to early K-type giants (with the exception of J150029.54+010744.8, which is a Ib-II supergiant), show carbon peculiarities in the form of weak $\mathrm{CH}$ (G-band) and CN bands.

\footnotetext{
${ }^{2}$ That library, as well as other MK standards libraries may be downloaded as ascii files (mklib.tar.gz) from http://www/appstate.edu/\$\sim\$grayro/mkclass. The MK standards used in libnor36 are listed on that same site.

${ }^{3}$ Note that the wavelengths used in this sub-section are those adopted by Grav \& Corballv (2009) and may differ slightly from the air wavelengths used elsewhere in the paper which are rounded to the nearest $\AA$.
} 


\section{RESULTS}

The final set of 8 stars that survived the vetting process described in the previous section are presented in Table 1. For completeness, we retain J150029.54+010744.8 in the set of candidates despite its luminosity class. A model atmosphere analysis will be needed to accurately place it relative to the giant branch. The table gives the date of observation of the measured SDSS DR10 spectrum, selected feature strengths and ratios as described in the text, the SDSS-assigned spectral type, and the spectral type determined by us. The $S(\operatorname{Li}$ I 6708$)$ values show that, despite the comparatively low resolution of the SDSS spectra, the absorption depths of the Li I lines in the candidates are not trivial, ranging from $5 \%$ to $17 \%$. The $D(\mathrm{Li}$ I 6708) values, the line depth in units of the local noise level, all suggest solid detections. Comparing the two columns of spectral types in the table, it is immediately apparent that our spectral types are all systematically earlier than the SDSS assignments. The differences are generally small and perhaps partially reflect the coarseness of the ELODIE library used by SDSS to classify the stars (Lee et al. 2008). Nuances introduced by weakening of $\mathrm{CH}$ and $\mathrm{CN}$ bands within a spectral type, captured by our approach and indicated in the "SpT Notes" column, might have confused the SDSS classification as well.

The DR7 dataset used by M\&S13 contains observations obtained up to July 2008 (Abazajian et al. 2009), whereas the DR10 dataset we used contains SDSS optical observations through June 2012 (Ahn et al. 2014). As we mentioned in Section 3.4.1, our approach captures all 9 M\&S13 stars in our dataset which have $S(\mathrm{Ca}$ I 4227) $\geq 0.4$. We note that Table 1 contains 4 additional stars that, according to their dates of observation, were present in the DR7 dataset. These stars apparently failed to pass one of the selection criteria used by M\&S13 to derive their list of 36 Li-rich candidates suitable for high resolution examination. It will be interesting to see whether or not future analysis of these stars confirms that they are Li-rich giants as we suggest. The spectra of the remaining 4 stars in Table 1 were obtained after July 2008 and could not have been considered by M\&S13. We find it somewhat surprising that we discovered only 4 new candidates among the stars observed after the end of the DR7 dataset. Our downloaded CHN17 dataset has 364,265 stars observed before July 2008 and 205,473 stars observed after that date. This makes the post-July 2008 portion of the dataset $56 \%$ of the size of the earlier portion. Given that we found a total of $13 \mathrm{Li}$-rich candidates in the earlier dataset (the $9 \mathrm{M} \& \mathrm{~S} 13$ stars plus our 4 new candidates), one naively might expect that the more recent portion alone would yield roughly 7 candidate Li-rich giants. That we found only 4 may be only a reflection of the uncertainty of small number statistics. It also may be the result of a shift in the spectral type mix between the two portions of the dataset given that the stellar classes targeted by the SDSS changed with time as the survey went on.

We show in Figure 2 spectra of the stars of Table 1 in the vicinity of the Li I line. We have

marked with dashed lines the Li I 6708 doublet, the Ca I 6718 feature used by Kumar et al. (2011) and used by us as a secondary criterion, and the TiO 6815 band head we used in Section 3.3, For comparison, we also show at the bottom the two high S/N M\&S13 stars with $S$ (Ca I 4227) $\geq 0.4$ having the weakest and the strongest Li I lines. It is apparent that the Li I features in our candidates are comparable in strength or stronger than those in stars identified as Li-rich giants by M\&S13. 


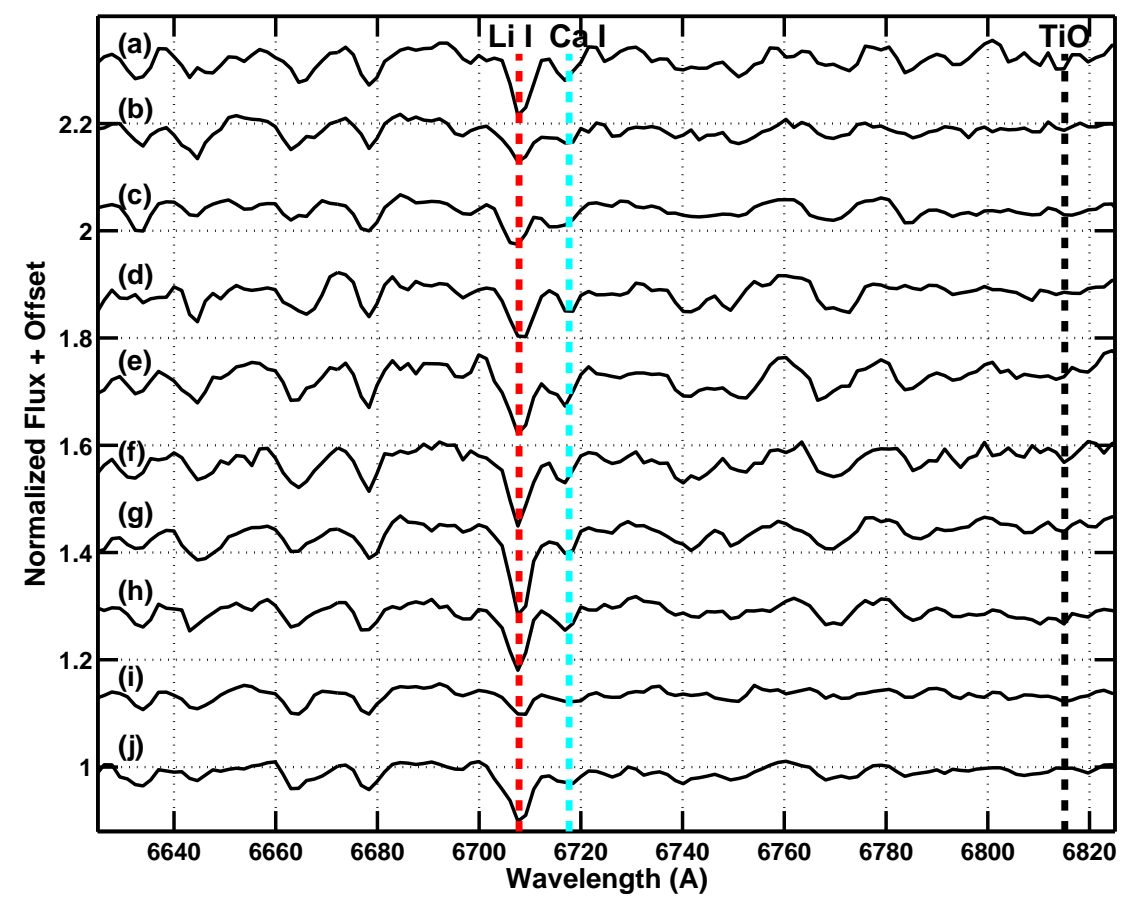

Fig. 2.- Comparison of the stars in Table 1 with two bounding M\&S13 stars. The spectra have been normalized to their mean flux in the interval [6693,6695]. The Table 1 stars are: (a) J021646.38-003333.5; (b) J060724.43+240052.4; (c) J062219.56+414403.8; (d) J092210.66+162455.9; $\quad$ (e) J122728.00+054420.2; $\quad$ (f) J143237.11+024533.4; (g) J150029.54+010744.8; (h) J183259.15+222243.5. The bottom two stars are the M\&S13 high S/N stars with the weakest and strongest Li I 6708 lines: (i) J030437.40+382346.1, and (j) J051523.18+155855.4, respectively. 
Finally, we show our Li-rich giant candidates in Figure 1 as green dots. Our 8 candidate Li-rich giants are distributed in the plot much like the 9 M\&S13 already-confirmed Li-rich giants. The most luminous are well away from the boundary between the MS and the post-MS stars. Like the majority of M\&S13 stars, the remainder of our candidates lie closer to the boundary. The locations of our candidates in Figure 1, their spectral types in Table 1, and their strong Li I lines (Figure 2) all suggest that they are Li-rich giants. We offer these candidates to researchers for closer examination, an undertaking well beyond the limited scope of this paper. Model atmosphere analyses of higher resolution spectra will be required to definitely determine the Li abundance and evolutionary status of our candidate Li-rich giants.

\section{SUMMARY}

In the current paper, we describe a new approach to identifying candidate Li-rich giants using the SDSS DR10 data release. As part of an earlier investigation (CHN17), 569,738 SDSS DR10 spectra were processed through a pipeline which yielded feature strength measurements for each of 1659 unique spectral features in each spectrum. The resulting nearly 2 billion feature measurements can be used to construct phase spaces of measurements. One may then introduce constraints that can be used to isolate stars with desired characteristics. In CHN17 this was accomplished using linked scatter plots and the hyperwall. In the current paper, we introduced a simple procedure for applying constraints that can be accomplished on a single workstation.

Guided by the literature on spectral classification of low resolution spectra, we searched for feature measurements that could be used to identify G and K giants, the subset of stars expected to harbor the Li-rich giants. Using the MILES spectra for MS and post-MS stars, we identified the $S$ (Ca I 4227) feature strength as the best surrogate for $T_{\text {eff }}$. After considerable experimentation, we found that the $S$ (Sr II 4078)/S(Fe I 4072) ratio gave reasonable separation between MS and post-MS stars for stars with $T_{\text {eff }} \lesssim 5800 \mathrm{~K}$. Armed with this insight, we isolated 1,523 potential Li-rich giants by applying constraints using only the feature strengths of Li I 6708, Ca I 4227, Sr II 4078, Fe I 4072, and $\mathrm{TiO} 6815$, as well as the median and local S/N values.

Visual inspection of the spectra quickly reduced the list of possible candidates to 49 stars, 9 of which were Li-rich giants already discovered by M\&S13. Next, the remaining 40 stars were systematically compared with those MILES dwarf and giant stars most similar to them in overall spectral energy distribution. Stars selected as most compatible with giants were then carefully classified for spectral type. These steps produced the 8 candidate Li-rich giants shown in Table 1 , We strongly recommend that researchers interested in expanding the list of known Li-rich giants consider these stars for detailed high resolution investigation.

The authors wish to thank Karen A. Huyser, Winifred M. Huo, and David W. Schwenke for 
their insightful comments on the draft of this paper. The authors also wish to thank the anonymous referee for numerous thoughtful suggestions that significantly improved the text. Funding for SDSS-III has been provided by the Alfred P. Sloan Foundation, the Participating Institutions, the National Science Foundation, and the U.S. Department of Energy Office of Science. The SDSS-III web site is http://www.sdss3.org/. SDSS-III is managed by the Astrophysical Research Consortium for the Participating Institutions of the SDSS-III Collaboration including the University of Arizona, the Brazilian Participation Group, Brookhaven National Laboratory, Carnegie Mellon University, University of Florida, the French Participation Group, the German Participation Group, Harvard University, the Instituto de Astrofisica de Canarias, the Michigan State/Notre Dame/JINA Participation Group, Johns Hopkins University, Lawrence Berkeley National Laboratory, Max Planck Institute for Astrophysics, Max Planck Institute for Extraterrestrial Physics, New Mexico State University, New York University, Ohio State University, Pennsylvania State University, University of Portsmouth, Princeton University, the Spanish Participation Group, University of Tokyo, University of Utah, Vanderbilt University, University of Virginia, University of Washington, and Yale University. This research has made use of the SIMBAD database, operated at CDS, Strasbourg, France. MATLAB(C) 2015 The MathWorks, Inc. MATLAB and Simulink are registered trademarks of The MathWorks, Inc. See www.mathworks.com/trademarks for a list of additional trademarks. Other product or brand names may be trademarks or registered trademarks of their respective holders.

Facilities: Sloan

\section{REFERENCES}

Abazajian, K. N., Adelman-McCarthy, J. K., Agüeros, M. A., et al. 2009, ApJS, 182, 543

Ahn, C. P., Alexandroff, R., Allende Prieto, C., et al. 2014, ApJS, 211, 17

Alcalá, J. M., Biazzo, K., Covino, E., Frasca, A., \& Bedin, L. R. 2011, A\&A, 531, L12

Boyajian, T. S., McAlister, H. A., van Belle, G., et al. 2012, ApJ, 746, 101

Brown, J. A., Sneden, C., Lambert, D. L., \& Dutchover, Jr., E. 1989, ApJS, 71, 293

Cameron, A. G. W., \& Fowler, W. A. 1971, ApJ, 164, 111

Carbon, D. F., Henze, C., \& Nelson, B. C. 2017, ApJS, 228, 19 [CHN17]

Casey, A. R., Ruchti, G., Masseron, T., et al. 2016, MNRAS, 461, 3336

Cenarro, A. J., Peletier, R. F., Sánchez-Blázquez, P., et al. 2007, MNRAS, 374, 664

Gray, D. F. 1992, The observation and analysis of stellar photospheres. (Cambridge, Great Britain: Cambridge University Press) 
Gray, R. O., \& Corbally, C. J. 2009, Stellar Spectral Classification (Princeton, New Jersey, U.S.A.: Princeton University Press)

-. 2014, AJ, 147, 80

Iben, Jr., I. 1967a, ApJ, 147, 624

-. 1967b, ApJ, 147, 650

Kirby, E. N., Guhathakurta, P., Zhang, A. J., et al. 2016, ApJ, 819, 135

Kraft, R. P., Peterson, R. C., Guhathakurta, P., et al. 1999, ApJ, 518, L53

Kumar, Y. B., Reddy, B. E., \& Lambert, D. L. 2011, ApJ, 730, L12

Lambert, D. L., \& Reddy, B. E. 2004, MNRAS, 349, 757

Lee, Y. S., Beers, T. C., Sivarani, T., et al. 2008, AJ, 136, 2022

Liu, Y. J., Tan, K. F., Wang, L., et al. 2014, ApJ, 785, 94

Mallik, S. V. 1999, A\&A, 352, 495

Martell, S. L., \& Shetrone, M. D. 2013, MNRAS, 430, 611 [M\&S13]

MATLAB. 2011, version 7.13.0.564 (R2011b) (Natick, Massachusetts: The MathWorks Inc.)

Prantzos, N., de Laverny, P., Guiglion, G., Recio-Blanco, A., \& Worley, C. C. 2017, A\&A, 606, A132

Reyniers, M., \& Van Winckel, H. 2001, A\&A, 365, 465

Ruchti, G. R., Fulbright, J. P., Wyse, R. F. G., et al. 2011, ApJ, 743, 107

Sánchez-Blázquez, P., Peletier, R. F., Jiménez-Vicente, J., et al. 2006, MNRAS, 371, 703

Wallerstein, G., \& Sneden, C. 1982, ApJ, 255, 577

White, R. J., Gabor, J. M., \& Hillenbrand, L. A. 2007, AJ, 133, 2524 
Table 1. Candidate Li-Rich Post-MS Stars

\begin{tabular}{|c|c|c|c|c|c|c|c|c|}
\hline Star Name & $\begin{array}{l}\text { Obs } \\
\text { Date }\end{array}$ & $\begin{array}{c}S \\
\text { Li I }\end{array}$ & $\begin{array}{c}D \\
\text { LI I }\end{array}$ & $\begin{array}{c}S \\
\mathrm{Ca} I\end{array}$ & $\begin{array}{l}\text { Sr II } \\
/ \mathrm{Fe} \mathrm{I}\end{array}$ & $\begin{array}{c}\text { SDSS } \\
\text { SpT }\end{array}$ & $\begin{array}{l}\text { Our } \\
\text { SpT }\end{array}$ & $\begin{array}{l}\text { SpT } \\
\text { Notes }\end{array}$ \\
\hline J021646.38-003333.5 & $10-10-2010$ & 0.14 & 10.7 & 0.68 & 1.64 & K4III & K0 III-IV & $\ldots$ \\
\hline $\mathrm{J} 060724.43+240052.4$ & $02-25-2008$ & 0.08 & 6.7 & 0.54 & 1.55 & $\mathrm{~K} 1$ & G9 III-IV & $\cdots$ \\
\hline J062219.56+414403.8 & $12-07-2007$ & 0.05 & 3.6 & 0.50 & 2.49 & K1 & G8 II-III & $\cdots$ \\
\hline J092210.66+162455.9 & 03-12-2012 & 0.11 & 6.6 & 0.64 & 4.40 & K4III & G9 II-III CN-1 & 1 \\
\hline $\mathrm{J} 122728.00+054420.2$ & 04-20-2009 & 0.14 & 10.6 & 0.85 & 1.14 & K5 & $\mathrm{K} 0$ III $\mathrm{CN}-1 \mathrm{CH}-1$ & 2 \\
\hline $\mathrm{J} 143237.11+024533.4$ & $03-31-2001$ & 0.15 & 8.2 & 0.77 & 4.25 & K5 & K0 II-III CN-2 CH-2 & 3 \\
\hline $\mathrm{J} 150029.54+010744.8$ & $05-19-2009$ & 0.17 & 12.7 & 0.61 & 4.73 & K5 & G8 Ib-II CN-1 & 4 \\
\hline $\mathrm{J} 183259.15+222243.5$ & $07-01-2006$ & 0.11 & 4.1 & 0.57 & 1.43 & K1 & G8 III CN-0.5 & 5 \\
\hline
\end{tabular}

${ }^{1} \mathrm{CN}$ band weak.

${ }^{2}$ Both $\mathrm{CN}$ and $\mathrm{CH}$ bands weak.

${ }^{3}$ Both $\mathrm{CN}$ and $\mathrm{CH}$ bands markedly weak.

${ }^{4} \mathrm{CN}$ band weak for luminosity type.

${ }^{5} \mathrm{CN}$ band slightly weak. 